\title{
Malignant Tracheal Invasion Masked by COVID-19 Symptoms
}

\author{
Chase $D$ Rose, MD', Andrew W Murray, $M D^{1}$, David G Lott, $M D^{2}$, Patrick A Devaleria, $M D^{3}$ and \\ Bradford B Smith, $M D^{1^{*}}$
}

\author{
${ }^{1}$ Department of Anesthesiology and Perioperative Medicine, Mayo Clinic, AZ, USA \\ ${ }^{2}$ Department of Otolaryngology-Head and Neck Surgery, Mayo Clinic, AZ, USA \\ ${ }^{3}$ Department of Cardiovascular and Thoracic Surgery, Mayo Clinic, AZ, USA
}

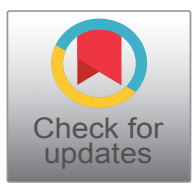

*Corresponding author: Bradford B Smith, MD, Department of Anesthesiology and Perioperative Medicine, Mayo Clinic, 5777 E. Mayo BLVD, Phoenix, AZ 85054, USA, Tel: 480-342-4313

\begin{abstract}
The coronavirus disease 2019 (COVID-19) pandemic has significantly altered the delivery of healthcare, particularly for patients undergoing perioperative surgical care. We present a case of successful airway and surgical management of a patient with substernal tracheal invasion by thyroid cancer whose care was complicated by a diagnosis of COVID-19. We recommend providers exhibit vigilance in addition to multidisciplinary evaluation and planning to limit exposure and avoid potential morbidity and mortality in this high risk patient population.
\end{abstract}

\section{Keywords}

Coronavirus disease 2019 (COVID-19), Tracheal invasion, Malignant thyroid carcinoma, Extracorporeal membrane oxygenation

\begin{abstract}
Abbreviations
SARS-CoV-2: Severe Acute Respiratory Syndrome Coronavirus 2; COVID-19: Coronavirus Disease 2019; CT: Computed Tomography; ECMO: Extracorporeal Membrane Oxygenation; HIPAA: Health Insurance Portability and Accountability Act; ASA: American Society of Anesthesiologist; V-V ECMO: Veno-venous Extracorporeal Membrane Oxygenation; ENT: Ear, Nose, and Throat; HEPA: High Efficiency Particulate Air
\end{abstract}

\section{Introduction}

Severe acute respiratory syndrome coronavirus 2 (SARS-CoV-2), the virus responsible for coronavirus disease 2019 (COVID-19), may produce a range of symptoms including fever, cough, or dyspnea and result in pneumonia or acute respiratory distress syndrome [1].
Perioperative care of COVID-19 patients is complicated by a high rate of pulmonary complications and mortality, in addition to the potential for provider transmission, particularly during aerosol generating procedures [2]. Preventative measures to protect patients and providers in an effort to reduce transmission have resulted in unfortunate delays in patient care, even in patients with known malignancy [3].

Invasive thyroid cancer may involve the trachea, esophagus, or strap muscles and carries a poor 10year survival rate ( 45\%) [4]. When the upper or lower airway is involved, patients may initially present with dyspnea or hoarseness and develop progressive respiratory compromise requiring surgical management. Thorough preoperative evaluation including computed tomography (CT) imaging, endoscopic airway evaluation, or ultrasound can be utilized to assess patient anatomy for airway and surgical planning purposes $[5,6]$. Previous case reports detailing airway management of patients with invasive thyroid masses describe awake fiberoptic intubation, awake tracheostomy, video laryngoscopy, laryngeal mask airway, high-frequency jet ventilation, and extracorporeal membrane oxygenation (ECMO) support as means to secure and/or support the airway until definitive surgical management is complete $[5,7]$. We present a case report of successful, multidisciplinary, perioperative management of a patient with substernal tracheal invasion by an unspecified neck mass whose care was complicated by a diagnosis of COVID-19. The patient provided The Health Insurance

Citation: Rose CD, Murray AW, Lott DG, Devaleria PA, Smith BB (2020) Malignant Tracheal Invasion Masked by COVID-19 Symptoms. Int J Anesthetic Anesthesiol 7:114. doi.org/10.23937/23774630/1410114

Accepted: November 25, 2020: Published: November 27, 2020

Copyright: (c) 2020 Rose CD, et al. This is an open-access article distributed under the terms of the Creative Commons Attribution License, which permits unrestricted use, distribution, and reproduction in any medium, provided the original author and source are credited. 

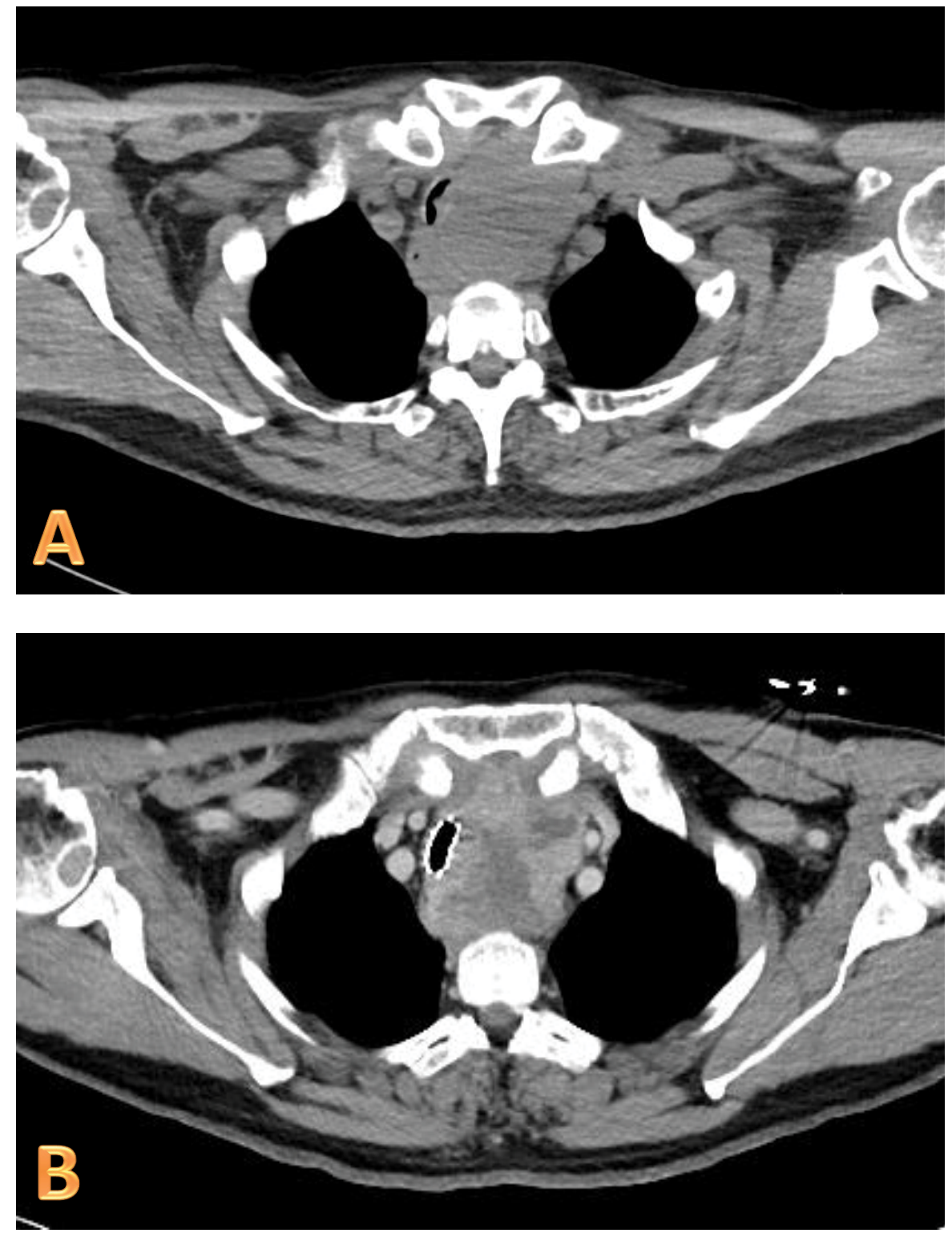

Figure 1: Computed tomography scan showing a large mass displacing and invading the lateral wall of the substernal trachea with a maximum residual airway diameter of 1.5 millimeters $(A)$ with interval tracheal stent placement $(B)$.

Portability and Accountability Act (HIPAA) authorization to publish this manuscript.

\section{Case Description}

A 65-year-old male presented to our institution with an unspecified neck mass. He was diagnosed with coronavirus disease 2019 (COVID-19) four-weeks prior at another institution after presenting for dyspnea. Following this initial diagnosis of COVID-19, he was instructed to self-quarantine at home without further evaluation into other possible etiologies for his dyspnea. He subsequently developed progressive dyspnea and orthopnea and again presented to an outside hospital. Repeat severe acute respiratory syndrome coronavirus 2 (SARS-CoV-2) testing was negative but a computed tomography (CT) scan revealed a large $(5 \times 5.5 \times 7.8$ $\mathrm{cm}$ ) mass arising from the left thyroid lobe, displacing and suggestive of tumor invading the lateral wall of the substernal trachea with a maximum residual airway diameter of 1.5 millimeters (Figure 1). He was transferred to our institution for urgent direct microlaryngoscopy, tracheal biopsy, and tracheal stent placement. Repeat SARS-CoV-2 testing was performed; the patient was maintained in isolation precautions and transferred to a negative pressure operating room. With the patient in a semi-recumbent position, standard ASA (American Society of Anesthesiologist) monitors were applied and titrated sedation with dexmedetomidine allowed for additional peripheral venous access and radial arterial line placement. Bilateral femoral venous sheaths were inserted by the cardiothoracic surgeon to allow for veno-venous extracorporeal membrane oxygenation (V-V ECMO) support in the event of sudden airway collapse. Difficult airway plan was discussed and appropriate equipment including fiberoptic bronchoscopes, rigid bronchoscope, and a tracheostomy tray were present. Titrated induction of anesthesia with dexmedetomidine and sevoflurane allowed for maintenance of spontaneous ventilation until it was determined that the patient could be mask ventilated. The patient was then 


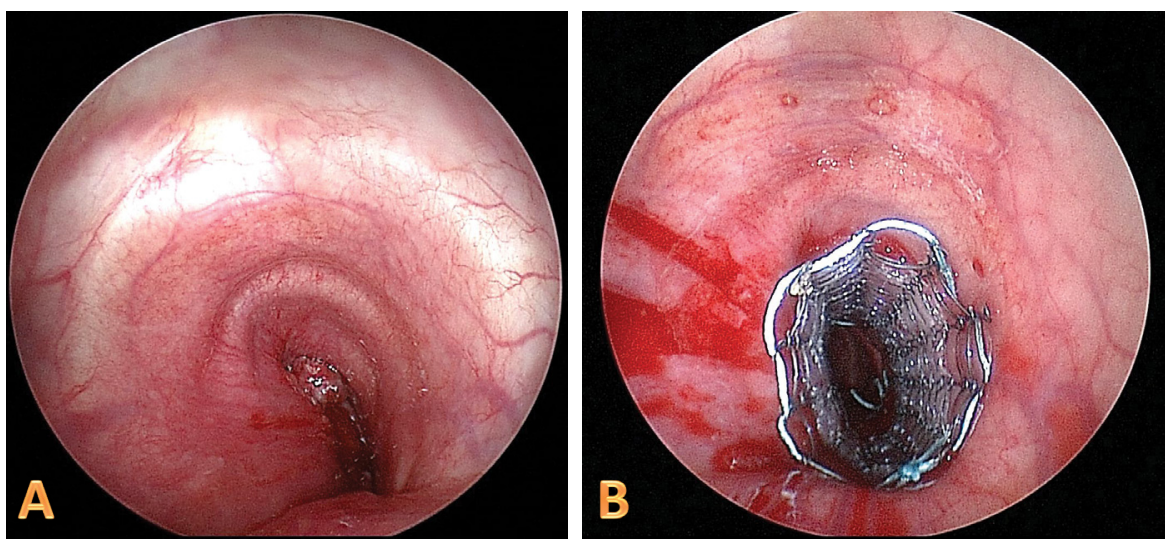

Figure 2: Intraoperative bronchoscopy showing tracheal lumen obstruction $(A)$ with subsequent tracheal stent deployment (B).

paralyzed and the ear, nose, and throat (ENT) surgeon performed direct laryngoscopy using a Dedo laryngoscope with suspension laryngoscopy. The airway was inspected and revealed significant mass effect, tumor invasion, and complete obstruction of the tracheal lumen (Figure 2A). A $6.5 \mathrm{~mm}$ Parker flexible endotracheal tube was able to be passed beyond the tumor and the patient was ventilated adequately. A balanced anesthetic was maintained throughout the case with dexmedetomidine, propofol, and fentanyl. There was difficulty with ventilation and progressive desaturation following tracheal biopsy. Bedside ultrasound revealed absent sliding lung over the left thorax. A flexible bronchoscope was used to evaluate the airway and a significant amount of blood was suctioned from the left main stem bronchus followed by bronchial lavage until adequate hemostasis was achieved, lung-sliding was confirmed, and hemodynamics improved. A $4 \mathrm{~cm}$ covered metal stent was then deployed at the area of stenosis and inflated to a diameter of $14 \mathrm{~mm}$ (Figure 2B). The patient was extubated in the operating room and transferred to an isolation bay in stable condition. The patient reported improvement in dyspnea immediately following the operation. Repeat SARS-CoV-2 testing returned the following day and was positive. Pathology revealed papillary thyroid cancer and the patient was discharged on postop day 3 with outpatient follow-up for initiation of chemoradiation.

\section{Discussion}

The COVID-19 pandemic has significantly altered the delivery of healthcare across the world. Measures to protect patients and providers in an effort to reduce transmission have resulted in delays in patient care, even in patients with known head and neck malignancy [3]. Widespread SARS-CoV-2 nasopharyngeal testing has been implemented to screen for and diagnose $\mathrm{CO}$ VID-19 in an effort to safely re-open all areas of society, including perioperative surgical care. Unfortunately, the SARS-CoV-2 reverse transcriptase polymerase chain reaction assay for oropharyngeal and nasopharyngeal te- sting has shown a high rate of false negative results [8]. In our patient, a diagnosis of COVID-19 was believed to be the etiology of his initial respiratory symptoms. Despite, persistent and progressive dyspnea and orthopnea, our patient avoided follow-up care for 1 month. At the time his neck mass was discovered, a negative SARS-CoV-2 test was reported at an outside facility and he was transferred to our institution. Per institutional policy, all patients transferring from outside facilities and all patients presenting for perioperative care must have COVID-19 testing performed at our facility. Given the patient's prior diagnosis of COVID-19 and persistent symptoms, he was treated as a suspected COVID-19 positive patient until repeat testing confirmed that his outside results were indeed false-positive. In urgent/emergent cases where COVID-19 status is unknown or the patient is suspected positive, all necessary precautions should be taken to limit provider and patient exposure.

The anesthetic management of this patient was complex and required multidisciplinary coordination. Given the patient's history COVID-19 positivity and nature of the high-risk aerosol-generating procedure, patient isolation and provider protection was paramount. The procedure was completed in a negative pressure operating room, only required personnel were present, all providers donned personal protective equipment consistent with droplet precautions (fitted N95 masks, face shields, gowns, gloves), a high efficiency particulate air (HEPA) viral filter was placed on the breathing circuit, a plastic drape covered the patient during emergence, and the patient was transported with a facemask to recover in a negative pressure isolation room.

The location and extent of tracheal invasion in this case required careful coordination with our ENT and cardiothoracic surgery colleagues to avoid potential respiratory collapse and mortality. Given the origin, size, and suspected or likely invasion of the substernal trachea by the patient's thyroid mass, options to secure the patient's airway, including laryngoscopy and elective/emergent tracheostomy were limited. We elected 
to maintain spontaneous ventilation with a titrated induction of anesthesia to determine if mask/positive pressure ventilation was possible prior to intubation. Additionally, femoral venous access was obtained to allow for $\mathrm{V}-\mathrm{V}$ ECMO support in the event that the patient's airway could not be secured or significant airway bleeding was encountered. Furthermore, since patients with COVID-19 who present for surgery have a high rate of pulmonary complications and mortality [2], it was unclear if the patient would tolerate intermittent apnea and require ECMO support until the tracheal stent could be deployed. While we were able to successfully mask ventilate and intubate the patient distally to the obstructive tumor, an individualized approach with multidisciplinary evaluation is necessary to avoid potential adverse events in this high risk population.

Invasive thyroid cancer may involve the upper or lower airway resulting in progressive respiratory compromise requiring surgical management. Thorough preoperative evaluation including CT imaging, endoscopic airway evaluation, or ultrasound can be utilized to assess patient anatomy for airway and surgical planning purposes $[5,6]$. While complete surgical resection is preferred, palliative stenting of the trachea can be attempted to allow for chemoradiation, as was completed in this case. Potential complications of tracheal stent deployment and/or tracheal biopsy include pneumothorax, bleeding, stent migration, mucostasis causing airway obstruction, and granuloma formation [9]. Unanticipated desaturation, elevated airway pressures, and difficulty with ventilation occurred in this case following tracheal biopsy. Point of care ultrasound, as described by Mittal and Gupta, was utilized to diagnose absent lung sliding over the left thorax at which time pneumothorax, hemothorax, and left mainstem occlusion were considered [10]. Flexible bronchoscopy revealed significant blood and mucus plugging the left mainstem bronchus which was sequentially cleared and resulted in restoration of sliding lung on ultrasound, resolution of elevated airway pressures, and improved hemodynamics. While the remainder of the procedure and postoperative care was uncomplicated, multidisciplinary coordination and discussion helped ensure a successful result for this patient.

\section{Conclusion}

Successful airway and surgical management of patients with invasive thyroid cancer often requires multi- disciplinary evaluation and thorough planning to ensure safe perioperative care. Additionally, providers must exhibit vigilance in the perioperative management of COVID-19 patients to limit exposure and avoid potential morbidity and mortality in this high risk patient population.

\section{Conflicts of Interest}

None.

\section{Financial Disclosures}

None.

\section{References}

1. Huang C, Wang Y, Li X, Ren L, Zhao J, et al. (2020) Clinical features of patients infected with 2019 novel corona virus in Wuhan, China. Lancet 395: 497-506.

2. Collaborative CO (2020) Mortality and pulmonary complications in patients undergoing surgery with perioperative SARS-CoV-2 infection: An international cohort study. Lancet 396: P27-P38

3. Werner MT, Carey RM, Albergotti WG, Lukens JN, Brody RM (2020) Impact of the COVID-19 pandemic on the management of head and neck malignancies. Otolaryngol Head Neck Surg 162: 816-817.

4. Price DL, Wong RJ, Randolph GW (2008) Invasive thyroid cancer: Management of the trachea and esophagus. Otolaryngol Clin North Am 41: 1155-1168.

5. Hohn A, Kaulins T, Hinkelbein J, Kaulina K, Kopp A, et al. (2017) Awake tracheotomy in a patient with stridor and dyspnoea caused by a sizeable malignant thyroid tumor: A case report and short review of the literature. Clin Case Rep 5: 1891-1895.

6. Rosenblatt W, lanus Al, Sukhupragarn W, Fickenscher A, Sasaki C (2011) Preoperative endoscopic airway examination (PEAE) provides superior airway information and may reduce the use of unnecessary awake intubation. Anesth Analg 112: 602-607.

7. Hoetzenecker K, Klepetko W, Keshavjee S, Cypel M (2017) Extracorporeal support in airway surgery. $\mathrm{J}$ Thorac Dis 9: 2108-2117.

8. Kucirka LM, Lauer SA, Laeyendecker O, Boon D, Lessler J (2020) Variation in false-negative rate of reverse transcriptase polymerase chain reaction-based SARS-CoV-2 tests by time since exposure. Ann Intern Med 173: 262-267.

9. Bhandari A, Wang YH, Lv SX, Xia EJ, Wang OC (2017) Novel strategy of stents in thyroid mass: A case series report of managing severely dyspneic patients. Onco Targets Ther 10: 4997-5004.

10. Mittal AK, Gupta N (2016) Intraoperative lung ultrasound: A clinicodynamic perspective. J Anaesthesiol Clin Pharmacol 32: 288-297.

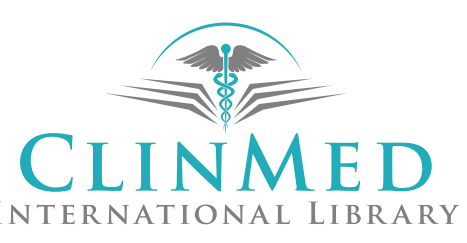

